\title{
L'influence de la carbonatation sur la biocolonisation de matériaux cimentaires dans le milieu marin
}

\author{
Mahmoud Hayek ${ }^{1, *}$, Marie Salgues ${ }^{1}$, Frédéric Habouzit ${ }^{2}$, Sandrine Bayle ${ }^{3}$, Jean-Claude Souche ${ }^{1}$, \\ Klartjee De Weerdt ${ }^{4}$, et Sylvain Pioch ${ }^{5}$ \\ ${ }^{1}$ LMGC, IMT Mines Alès, Univ Montpellier, CNRS, 6, avenue de Clavières, 30319 Alès Cedex, France \\ 2 INRA, UR0050, Laboratoire de Biotechnologie de l'Environnement (LBE), 102, avenue des Etangs, F11100 Narbonne, France \\ ${ }^{3}$ IMT Mines Alès, Laboratoire de génie de l'environnement Industriel (LGEI), 6, avenue de Clavières, 30319 Alès Cedex, France \\ ${ }^{4}$ Norwegian University of Science and Technology NTNU, Department of Structural Engineering, Trondheim, Norway \\ ${ }^{5}$ UMR 5175 CEFE, Université Paul Valéry, Montpellier, SupAgro, EPHE, Laboratoire Biologie Ecologie Environnement-UPV, \\ Route de Mende, 34000 Montpellier, France
}

Reçu le 3 février 2020 / Accepté le 8 juillet 2020

\begin{abstract}
Résumé. Les ingénieurs spécialisés en génie civil doivent intégrer dans leur design des mesures de protection de la biodiversité marine de qui autorise le concept d'écoconception d'infrastructures marines respectueuses de l'environnement. Les matériaux cimentaires sont privilégiés pour concevoir les infrastructures marines et interagissent avec le milieu marin. Ces structures seront colonisées par des micro-organismes et macroorganismes marins. Dans un premier temps, les organismes forment un biofilm à la surface du béton en fonction des facteurs environnementaux et des propriétés physico-chimiques du matériau. Dans cette étude, il a été démontré que la pré-carbonatation des matériaux cimentaires accélère la croissance des micro-organismes formant le biofilm à leur surface en abaissant le $\mathrm{pH}$ de la surface. Il a été démontré en laboratoire que la colonisation de la surface par des micro-organismes augmente également l'hydrophobicité de la surface et pourrait ainsi améliorer la durabilité du matériau en diminuant l'absorption d'eau de mer contenant des agents agressifs.
\end{abstract}

Mots clés : biocolonisation / biofilm / environnement marin / matériaux cimentaires / écologie / in vitro tests

\begin{abstract}
The influence of carbonation on the biocolonization of cementitious materials in the marine environment. Engineers specializing in civil engineering must integrate into their design measures to protect marine biodiversity, which authorizes the concept of eco-design of marine infrastructure that respects the environment. The cementitious materials are preferred to design marine infrastructure and interact with the marine environment. These structures will be colonized by marine microorganisms and macroorganisms. Initially, the organisms form a biofilm on the concrete surface according to environmental factors and the physicochemical properties of the material. In this study, it was shown that the pre-carbonation of cementitious materials accelerates the growth of microorganisms forming a biofilm by lowering the $\mathrm{pH}$ of the surface. It was shown using a laboratory test that colonization of the surface by microorganisms also increases the hydrophobicity of the surface and could thus improve the durability of the material by reducing the absorption of seawater containing aggressive ions.
\end{abstract}

Keywords: biocolonization / biofilm / marine environment / cementitious materials / marine ecology / in vitro tests

\section{Introduction}

Les matériaux cimentaires sont des matériaux essentiels dans les différents domaines de la construction: ouvrages d'art, génie civil et génie maritime comme les ports maritimes et les ouvrages côtiers [1]. La durabilité de ces matériaux cimentaires dépend fortement des conditions

\footnotetext{
*e-mail: mahmoud.hayek@mines-ales.fr
}

environnementales auxquelles ils sont exposés. Ainsi, les ingénieurs qui conçoivent des ouvrages de génie civil doivent d'abord effectuer des investigations intensives pour assurer une meilleure durée de vie des ouvrages tout en respectant les contraintes économiques. En milieu marin, le vieillissement des matériaux cimentaires est lié à une multitude d'attaques de natures différentes (physiques, chimiques et biologiques) qui peuvent être agressives envers le matériau et agir en synergie, conduisant à la détérioration de la structure [2]. Bien que les attaques de 
nature mécanique et physico-chimique soient généralement bien comprises pour optimiser la durabilité des matériaux cimentaires et soient soumises à des normes et recommandations, celles qui sont de nature biologique sont souvent négligées. Cependant, il convient de noter que la durabilité du matériau et les interactions biologiques entre le matériau et l'environnement sont connectées [3-5].

Dans l'environnement marin, tous les substrats vivants ou morts, naturels ou artificiels, sont rapidement colonisés par les micro-organismes et les macroorganismes présents naturellement dans l'eau de mer [6-9]. Cette biocolonisation peut être divisée en deux étapes principales, le microfouling et le macrofouling, qui se caractérisent respectivement par la formation de biofilm microbien et par l'adhésion de macroorganismes tels que les algues, les bernacles et les larves (macrofouling).

Dans les minutes qui suivent l'immersion d'un substrat, les molécules et les particules organiques sont adsorbées à la surface, qui est ensuite colonisée par des bactéries qui forment un biofilm. Les biofilms bactériens sont composés d'une ou de plusieurs espèces de bactéries fixées au substrat (et entre elles) et enfermées dans une matrice de substances polymériques extracellulaires (EPS) [10]. Les différentes étapes de la formation de biofilm bactérien sont: i) l'adhésion réversible et irréversible des bactéries à la surface du matériau cimentaire, ii) la croissance et la maturation du biofilm avec la sécrétion d'EPS, iii) le détachement partiel et la dispersion des cellules bactériennes. Les biofilms bactériens matures ont des structures tridimensionnelles complexes, qui dépendent de la composition spécifique du biofilm, de l'activité bactérienne et des conditions environnementales surtout l'hydrodynamisme du milieu [11-13]. Le biofilm bactérien est le premier colonisateur qui facilite l'adhésion d'autres organismes tels que les champignons, les microalgues, les macroalgues et les invertébrés formant ainsi un biofouling [14-16].

La biocolonisation des structures maritimes (briselames, les digues, les jetées, les quais, les structures offshore et les écluses...) est le point de départ de différentes interactions biologiques (biodégradation, bioprotection...) pouvant avoir lieu entre les matériaux cimentaires et la vie marine. La biodégradation du béton se caractérise par l'endommagement de la structure (les propriétés du matériau, sa composition, ses caractéristiques mécaniques peuvent être modifiées) par les activités des organismes vivants $[17,18]$. Les micro-organismes affectent la stabilité du béton en contribuant à l'érosion de sa surface, ce qui augmente la porosité du béton et favorise la pénétration d'ions agressifs $\left(\mathrm{Cl}^{-}, \mathrm{Mg}^{2+}, \mathrm{OH}^{-}\right)$qui peuvent générer ou accélérer l'endommagement du béton (fissuration, écaillage...) [19]. En revanche, la biocolonisation peut également protéger le béton colonisé au moyen d'une couche protectrice d'organismes et d'EPS, barrière physique, qui réduit la perméabilité de la surface, conduisant à une meilleure durabilité du matériau (bioprotection) [20-25].

La compréhension des interactions entre les microorganismes et les matériaux cimentaires est donc cruciale et constitue une étape fondamentale vers des structures plus durables, plus sûres et de meilleure qualité dans de nombreux contextes. En outre, il a été mentionné dans la littérature que la bioréceptivité du matériau (capacité à être colonisé par des organismes vivants) est déterminée par la nature et les propriétés physicochimiques de la surface [26,27] : la composition chimique [28,29], la rugosité [30,31], la porosité [32-34], l'hydrophobicité [29,35-37], et le $\mathrm{pH}[4,33]$.

En milieu marin, des études complémentaires sont nécessaires pour déterminer les différents facteurs pouvant influencer la biocolonisation et donc la bioreceptivité des matériaux cimentaires. À la lumière de ce manque d'informations concernant les facteurs qui agissent sur la biocolonisation de matériaux cimentaires en milieu marin, l'objectif de cette étude est d'étudier l'influence du $\mathrm{pH}$ de surface et de l'hydrophobicité sur la biocolonisation des matériaux cimentaires dans des conditions contrôlées. L'objectif à plus long terme de cette étude est de développer une approche expérimentale qui pourrait aider les ingénieurs civils à concevoir des structures marines en spécifiant le type et les caractéristiques physico-chimiques des matériaux cimentaires à utiliser afin d'écoconcevoir les structures maritimes.

\section{Matériaux et méthodes}

\subsection{Formulation des matériaux cimentaires}

Deux types de matériaux cimentaires ont été utilisés (bétons et pâtes de ciment) afin d'étudier l'effet de la biocolonisation sur les propriétés physico-chimiques des matériaux cimentaires. Les échantillons de béton utilisés dans cette étude ont été extraits de disques de béton (diamètre de $11 \mathrm{~cm}$ et hauteur de $7 \mathrm{~cm}$ ) déjà préparés dans le cadre d'un projet précédent (en 2016 par Souche et al.) [30,31]. La composition des disques comprend un ciment Portland CEM I 52,5 N PM ES, un sable silico calcaire recomposé, un gravier silico-calcaire naturel, ainsi qu'un super-plastifiant haut réducteur d'eau avec un rapport eau sur ciment de 0,6. Les compositions de ces échantillons sont détaillées dans le tableau 1 tableau 1 (désignés par la lettre B) de la publication de Souche et al., en 2016 [31]. Afin d'obtenir les échantillons de béton utilisés dans cette étude, ces disques en béton ont été carottés avec une couronne de $2,2 \mathrm{~cm}$ puis sciés pour obtenir des échantillons de $2,2 \mathrm{~cm}$ de diamètre et de $0,3 \mathrm{~cm}$ de haut. Chaque échantillon de béton ainsi obtenu après sciage a été observé et sélectionné pour être représentatif (présence de pâte et de granulats).

De plus, des échantillons composés de pâte de ciment Portland ont également été préparés en mélangeant du ciment Portland et de l'eau dans un rapport $\mathrm{E} / \mathrm{C}$ de 0,5 . Le même type de ciment Portland CEM I (CEM I 52,5 N PM ES) utilisé dans la préparation de béton a été choisi pour préparer les échantillons de pâte cimentaire. Après mélange, la pâte de ciment a été coulée dans des moules cylindriques de $2,2 \mathrm{~cm}$ de diamètre et $2 \mathrm{~cm}$ de hauteur et ont été conservées 7 jours à $20^{\circ} \mathrm{C}$. Les échantillons de pâte de ciment ont été ensuite démoulés et placés à $20^{\circ} \mathrm{C}$ à l'air pendant 7 jours avant d'être immergés dans de l'eau de mer en laboratoire.

Plusieurs tests ont été effectués sur ces matériaux notamment dans des Erlenmeyers, ce qui justifie la nécessité de travailler avec des échantillons de faibles dimensions. Afin de faciliter l'évaluation de l'angle de 

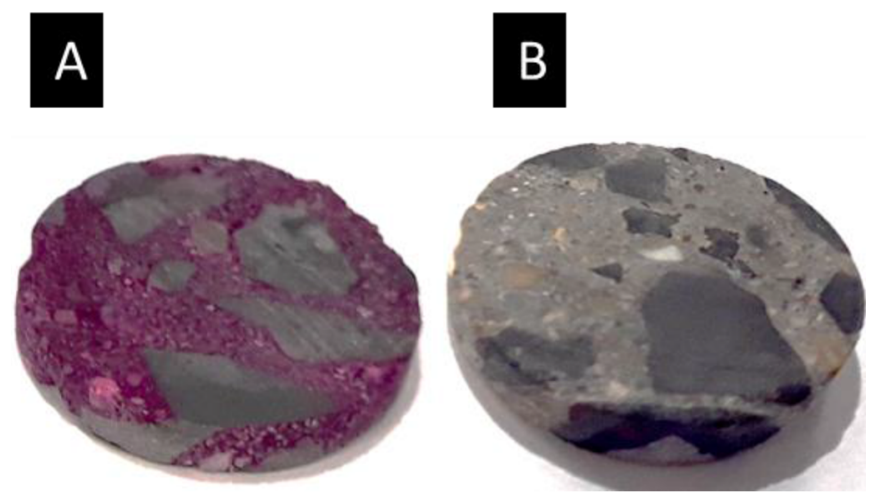

Fig. 1. Test de phénolphtaléine sur la surface des disques du béton obtenus après sciage. (A) disque prélevé au cœur de l'échantillon cylindrique $11 \times 7 \mathrm{~cm}$. (B) disque prélevé de l'extrémité de l'échantillon cylindrique $11 \times 7 \mathrm{~cm}$.

Fig. 1. The phenolphthalein tests on the surface of the concrete discs obtained after sawing. (A) Disc taken from the heart of the $11 \times 7 \mathrm{~cm}$ cylindrical sample. (B) Disc taken from the end of the $11 \times 7 \mathrm{~cm}$ cylindrical sample.

contact des échantillons avec l'eau de mer, la pâte de ciment a été privilégiée dans le but de travailler avec une surface plus homogène qui ne contient pas de granulats, ce qui rend plus fiables les valeurs de l'angle de contact mesurées.

Un test à la phénolphtaléine a mis en évidence que les disques sciés à l'extrémité du cylindre sont carbonatés en surface, ce qui n'est pas le cas des disques prélevés au cour de l'éprouvette (Fig. 1).

\subsection{Quantification de la biocolonisation}

Un test de biocolonisation en laboratoire a été effectué sur les échantillons de béton et de pâte de ciment. Dans les deux cas, le milieu utilisé était de l'eau de mer récupérée de la station IFREMER de Palavas (Unité de recherche biologique pour les organismes marins exploités) dans des bouteilles en verre stériles (autoclave, $121^{\circ} \mathrm{C}$ pendant 15 minutes). Dans le cas du béton, deux types d'échantillons ont été utilisés, le béton carbonaté et le béton non carbonaté, tandis que dans le cas de la pâte de ciment, la variable était le milieu utilisé ; eau de mer naturelle et eau de mer stérile (eau de mer naturelle stérilisée en autoclave) (Fig. 2).

Pour réaliser ce test, les échantillons de béton ou de pâte de ciment ont été stérilisés par autoclave à $121^{\circ} \mathrm{C}$ pendant 15 minutes puis ils ont été déposés au fond des Erlenmeyers stériles de $250 \mathrm{ml}$ contenant $50 \mathrm{ml}$ d'eau de mer (4 échantillons / Erlenmeyer) [21]. Les Erlenmeyers ont ensuite été incubés à $20^{\circ} \mathrm{C}$ et $80 \mathrm{rpm}$ (rotation par minute) pour assurer l'oxygénation du milieu (nécessaire à la croissance des micro-organismes). Après chaque période d'incubation, la surface des échantillons est rincée doucement trois fois avec $1 \mathrm{ml}$ d'eau de mer stérile pour éliminer les microorganismes non adhérents de sa surface. Les échantillons sont ensuite placés dans des tubes stériles contenant $5 \mathrm{ml}$ d'eau de mer stérile. Les tubes sont plongés pendant 10 minutes à $20^{\circ} \mathrm{C}$ dans un bain à ultrasons (Bandelin
SONOREX ${ }^{\mathrm{TM}}$ ) afin de détacher les micro-organismes adhérents à la surface de l'échantillon. L'eau de mer présente dans les tubes est récupérée et étalée sur des boîtes de Pétri contenant du milieu de culture dédié à la croissance des bactéries marines, le Marine Agar (Difco ${ }^{\mathrm{TM}}$ Marine Agar 2216). Ces boîtes ont ensuite été incubées à $20^{\circ} \mathrm{C}$ et le comptage des colonies est réalisé $72 \mathrm{~h}$ après l'étalement. Les résultats sont exprimés en unités formant colonie par $\mathrm{cm}^{3}$ de béton ou de pâte de ciment $\left(\mathrm{UFC} / \mathrm{cm}^{3}\right)$.

\subsection{Mesure de $\mathrm{pH}$}

Les mesures de $\mathrm{pH}$ de l'eau de mer et de la surface des matériaux cimentaires ont été effectuées en utilisant respectivement une électrode de $\mathrm{pH}$ (instrument Hanna, HI1230) et du papier indicateur de $\mathrm{pH}$ (Whatman, 0,0 à 14,0). Dans le cas de la mesure du $\mathrm{pH}$ de l'eau de mer, l'électrode de $\mathrm{pH}$ a été soigneusement rincée à l'eau distillée avant d'être plongée dans une eau de mer bien agitée $(30 \mathrm{ml})$. La valeur du pH a été notée lorsque la lecture du pH est stable. Dans le cas des mesures en surface des matériaux cimentaires, un millilitre d'eau ultra pure (eau Milli-Q) est apposé sur la surface de l'échantillon. Après une minute, le papier $\mathrm{pH}$ a été déposé sur la surface et la valeur du $\mathrm{pH}$ a été évaluée après $30 \mathrm{~s}$ de contact entre le papier $\mathrm{pH}$ et la surface $[32,33]$. Cette méthode permet d'évaluer le $\mathrm{pH}$ de la surface et non le $\mathrm{pH}$ du matériau complet. Il est à noter que ce pH évalué peut être influencé par le biofilm potentiel présent à la surface.

\section{4 Évaluation de l'hydrophobicité de surface}

L'hydrophobicité des matériaux cimentaires a été évaluée à l'aide d'un analyseur de forme de goutte (KRÜSS, DSA 30) qui mesure les angles et les diamètres de contact entre une goutte et une surface [38]. Après chaque incubation dans l'eau de mer, une goutte d'eau $(3 \mu \mathrm{L})$ a été déposée à la surface des matériaux cimentaires et le suivi de son absorption a été effectué à l'aide d'une caméra (8 images par seconde). Les angles de contact entre la goutte et le matériau ont été mesurés à l'aide du logiciel de la machine Advance. Un minimum de trois tests est réalisé en différents points et les résultats sont présentés sous forme d'évaluation de l'angle de contact $\left(^{\circ}\right)$ en fonction du temps (jour).

\section{Résultats}

\subsection{Test en laboratoire sur les échantillons de béton}

Afin d'étudier l'influence de la carbonatation sur la biocolonisation de la surface du béton en milieu marin, des échantillons de béton dit «carbonaté » ( $\mathrm{pH}$ inférieur à 9 ) et dit «non carbonaté » ( $\mathrm{pH}$ autour de 10-11) ont été incubés en laboratoire à $20^{\circ} \mathrm{C}$ et $80 \mathrm{rpm}$ dans l'eau de mer naturelle. Le biofilm formé sur la surface du béton a été quantifié après $0,1,3,7,10,15,24,29$ et 60 jours (Fig. 3A). De même, le $\mathrm{pH}$ de la surface du béton (Fig. 3A) et le $\mathrm{pH}$ de l'eau de mer ( $\mathrm{pH}$ de la solution d'exposition) (Fig. 3B) ont été suivis et mesurés. 


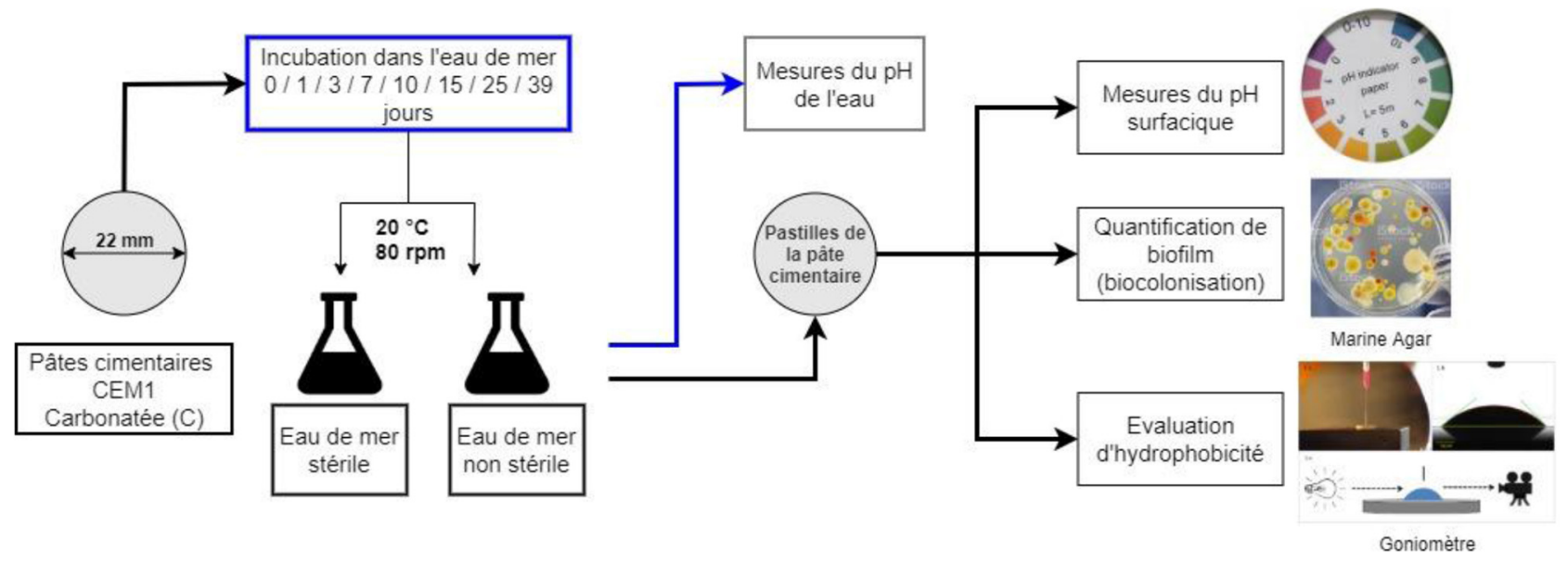

Fig. 2. Démarche expérimentale du test de biocolonisation effectué au laboratoire.

Fig. 2. Experimental approach of the biocolonization laboratory test.

A

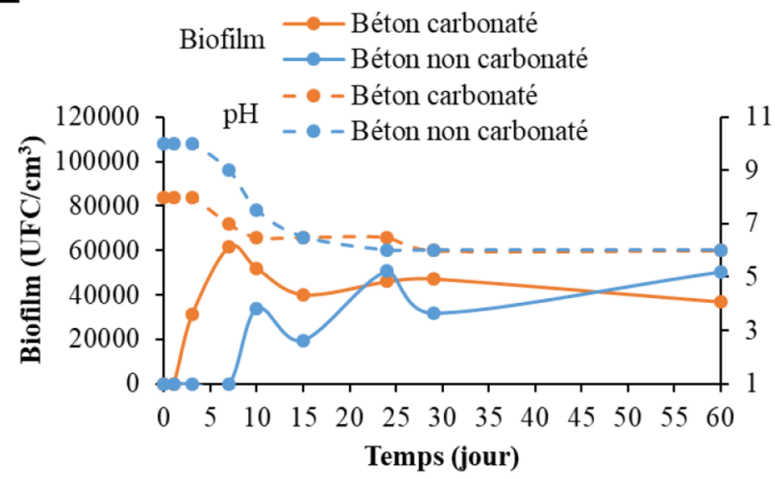

B

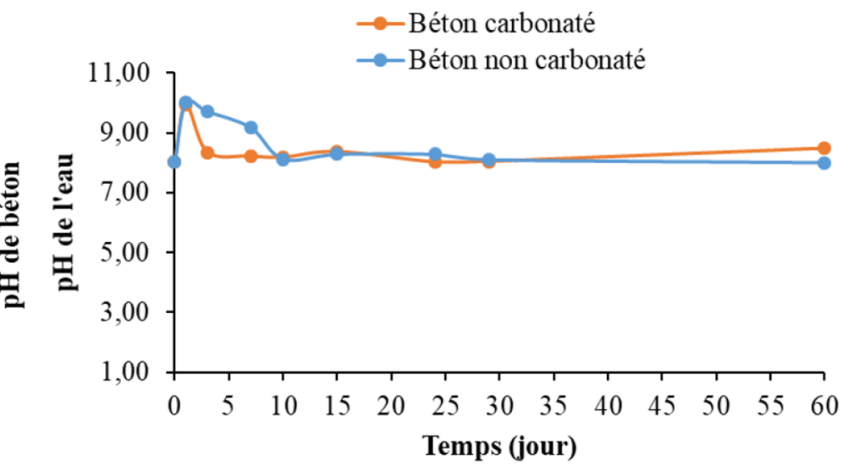

—Béton carbonaté $\quad$ Béton non carbonaté

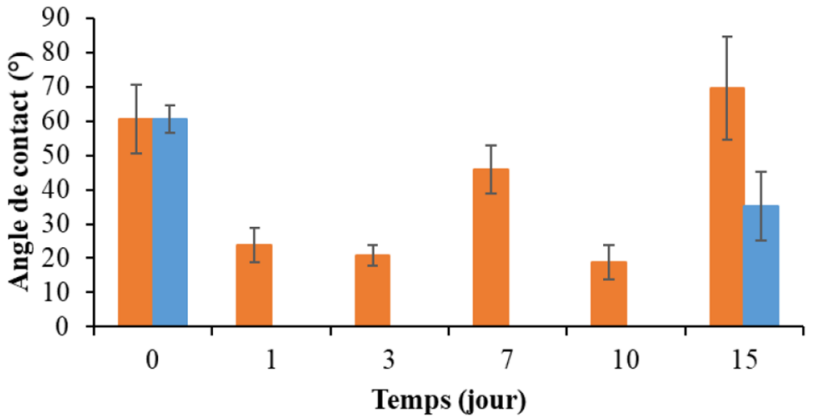

Fig. 3. Résultats obtenus avec le test de biocolonisation en utilisant des échantillons de béton. (A) Quantification du biofilm et évaluation du pH de la surface du béton. (B) Évaluation du pH de l'eau contenant les échantillons carbonatés et non carbonatés. (C) Valeurs d'angle de contact d'une goutte d'eau déposée sur la surface de l'échantillon (chaque expérience a été réalisée en triplicat).

Fig. 3. Results obtained with the biocolonization laboratory test using concrete samples. (A) Quantification of the biofilm and evaluation of the $\mathrm{pH}$ of the concrete surface. (B) Evaluation of the $\mathrm{pH}$ of seawater containing the carbonated and non-carbonated samples. (C) Contact angle values of a drop of water deposited on the surface of the sample (each experiment was carried out in triplicate). 
Les résultats montrent que la formation de biofilm sur les échantillons carbonatés et non carbonatés a débuté par une phase de latence suivie d'une phase de croissance et d'accumulation des cellules sur la surface. Cette cinétique de colonisation a également été observée par Tran et al. lors d'essais de colonisation in vitro et in situ sur des échantillons de mortier $[32,33]$. Dans le cas d'échantillons carbonatés, la formation de biofilm est rapide avec une phase de latence quasi inexistante. L'accumulation de biofilm à la surface atteint un maximum après 7 jours d'incubation puis diminue légèrement pour atteindre une phase de plateau. Cependant, dans le cas d'échantillons non carbonatés, une phase d'induction de 7 jours est observée et la formation de biofilm atteint un maximum après 24 jours d'incubation avec une valeur proche à celle des échantillons carbonatés (Fig. 3A). La différence de période de latence entre ces deux cas peut être expliquée par les pH et les phases minérales (tels que l'apparition de carbonate de calcium suite à la réaction de carbonatation) de surface différents à T0 des échantillons carbonatés ( $\mathrm{pH}$ autour de 8) et non carbonatés (pH supérieur à 10). Cette influence de la carbonatation de surface a également été identifiée dans plusieurs études concernant la colonisation de matériaux cimentaires. Ces études ont montré une phase de latence plus élevée dans le cas d'échantillons non carbonatés [32,33,39]. De même, Dooley et al. (1999), Guilbeau et al. (2003), Prieto et al. (2004) ont montré que la carbonatation favorise l'attachement et la croissance des micro-organismes (algues) lors d'essais de laboratoire [4042].

Une diminution du $\mathrm{pH}$ de surface semble nécessaire pour le développement du biofilm sur des échantillons non carbonatés; la croissance du biofilm débute après 5 jours lorsqu'une diminution du pH de surface de 10 (T0) à 9 (T5) est observée. Le pH de l'eau de mer contenant les échantillons carbonatés est resté à 8 tout au long de l'expérience, ce qui a permis une croissance normale des micro-organismes à la surface du béton (Fig. 3B). Dans le cas d'échantillons non carbonatés, le pH de l'eau de mer augmente à 10 en raison notamment de la libération de $\mathrm{Ca}$ $(\mathrm{OH})_{2}$ et de $\mathrm{KOH}$ dans l'eau de mer, puis diminue à 8 (neutralisation des bases reléguées dans l'eau) après le septième jour d'incubation (fin de la phase de latence). Ces résultats indiquent que la carbonatation joue un rôle primordial dans l'initiation de la biocolonisation du béton. Le test en laboratoire fonctionne en circuit fermé ce qui indique que le $\mathrm{pH}$ de la suspension est sous l'influence de la composition chimique de béton utilisé notamment le relargage des ions hydroxydes suite à l'attaque de l'eau de mer. La valeur du pH de l'eau dans le cas d'échantillons non carbonatés est supérieure à celle dans le cas d'échantillons carbonatés, ce qui ralentit la colonisation des échantillons non carbonatés par rapport aux échantillons carbonatés.

Afin d'évaluer l'effet de la formation de biofilms sur les caractéristiques physico-chimiques du béton, l'interaction de la surface du béton avec l'eau a été examinée en mesurant l'angle de contact d'une goutte d'eau déposée sur la surface de l'échantillon (Fig. 3C). Cet examen a été réalisé sur des échantillons incubés dans l'eau de mer entre
0 et 15 jours. Après 15 jours d'incubation, un dépôt de sel formé à la surface de l'échantillon rend impossible la mesure de l'angle de contact.

À T0, les échantillons de béton carbonaté et non carbonaté ont montré un comportement similaire, car l'angle de contact dans les deux cas était d'environ 60 degrés, ce qui est relativement élevé [43]. Après incubation dans l'eau de mer, l'angle de contact des échantillons carbonatés diminue puis augmente à nouveau à T15 pendant la phase de plateau du développement du biofilm. Dans le cas d'échantillons non carbonatés, après immersion, l'angle de contact devient nul jusqu'à T15. Pendant cette période, la goutte d'eau déposée sur la surface est rapidement absorbée par l'échantillon et répartie sur toute la surface. À T15, une valeur de 35 degrés a été mesurée. Cette régénération de l'angle de contact pourrait s'expliquer par la formation de biofilm à la surface des échantillons. La matrice extracellulaire du biofilm pourrait alors protéger le matériau cimentaire en diminuant l'absorption d'eau contenant des substances agressives.

En résumé, cette étude a montré que la pré-carbonatation du béton accélère le développement des microorganismes à sa surface puisque la phase de latence est réduite dans le cas d'échantillons carbonatés. La colonisation de la surface conduit à un angle de contact plus élevé indiquant une surface plus hydrophobe qui peut potentiellement protéger le matériau en diminuant l'absorption d'eau contenant des substances agressives.

\subsection{Test en laboratoire sur les pâtes de ciment}

Les résultats obtenus avec le test précédent en utilisant des échantillons de béton ont suggéré une relation probable entre la concentration des bactéries présentes sur la surface (le biofilm) et la valeur de l'angle de contact de cette surface avec l'eau (''hydrophobicité). Pour valider cette relation et être sûr que l'amélioration de l'angle de contact avec l'eau est provoquée par la colonisation de la surface et non par l'interaction chimique avec l'eau de mer, les mêmes tests de laboratoire ont été réalisés sur pâtes cimentaires immergées dans de l'eau de mer naturelle et stérile (pas de microorganismes). Les valeurs de l'angle de contact obtenues avec ce test sont plus fiables grâce à la surface homogène et à la nature de la pâte de ciment qui absorbe plus que les granulats contenus dans le béton.

Avant les essais, les pâtes de ciment utilisées ont été placées dans une chambre aérée pendant 7 jours pour obtenir des échantillons de pâte carbonatée. Cependant, la mesure du $\mathrm{pH}$ de surface préalablement aux tests montre un pH très basique (valeur égale à 10) (Fig. 4A). Ce pH a également été vérifié à l'aide d'un indicateur de phénolphtaléine qui a donné une coloration rose après contact avec la surface de la pâte de ciment.

La biocolonisation et les angles de contact des échantillons de pâte de ciment ont été étudiés pendant 34 jours dans de l'eau de mer naturelle et stérile (Fig. 4). Ce test a été effectué en circuit fermé ce qui indique que le pH de la suspension est sous l'influence de la composition chimique de pâte de ciment utilisée notamment le relargage 
A

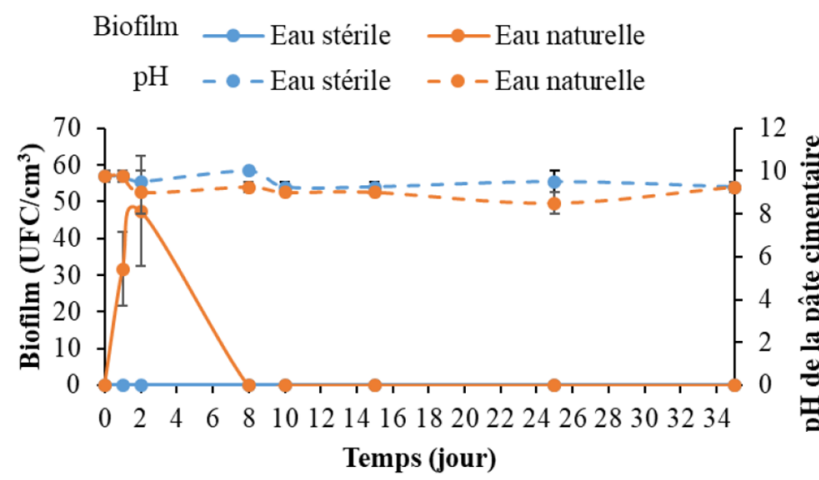

B

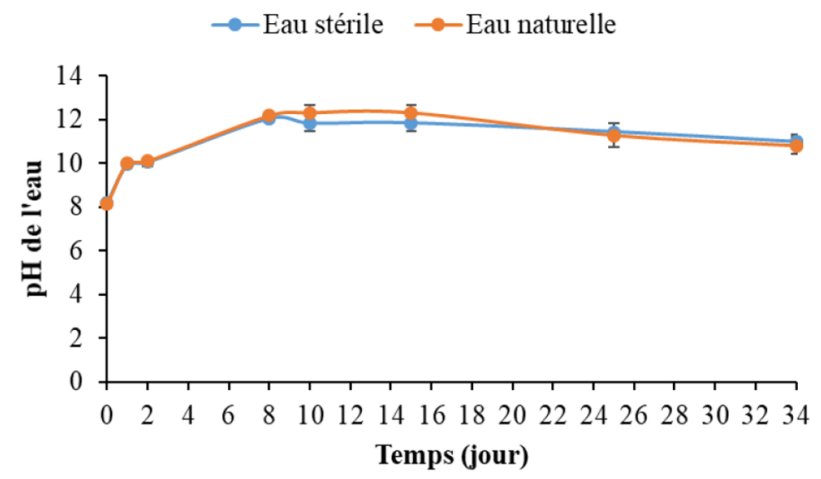

C

Eau stérile Eau naturelle

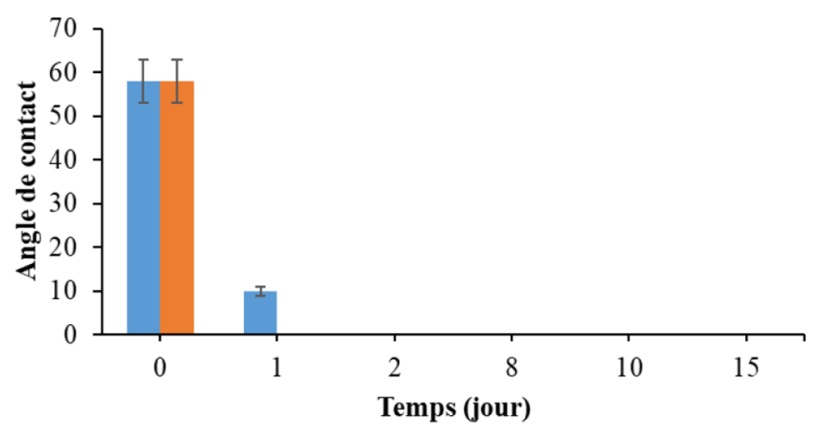

Fig. 4. Résultats obtenus avec le test de biocolonisation en utilisant des échantillons de pâte de ciment. (A) Quantification du biofilm et évaluation du pH de la surface de la pâte de ciment. (B) Évaluation du pH de l'eau contenant les échantillons de pâte. (C) Angle de contact Valeurs d'une goutte d'eau déposée sur la surface de l'échantillon. (Chaque expérience a été réalisée en triplicat).

Fig. 4. Results obtained with the biocolonization laboratory test using cement paste samples. (A) Quantification of the biofilm and evaluation of the $\mathrm{pH}$ the cement paste surface. (B) Evaluation of the $\mathrm{pH}$ of seawater containing the samples. (C) Contact angle values of a drop of water deposited on the surface of the sample. (Each experiment was carried out in triplicate).

des ions hydroxydes suite à l'attaque de l'eau de mer. La valeur du pH de l'eau dans ce cas est de l'ordre de 12 (Fig. $4 \mathrm{~B})$, valeur très basique qui inhibe complètement la croissance des micro-organismes [44]. En raison de ce $\mathrm{pH}$ basique, aucune biocolonisation des échantillons n'est observée tout au long de l'expérience (Fig. 4A).

Cependant, les données d'angle de contact montrent que l'absence de biocolonisation conduit à une absorption rapide de la goutte d'eau. Aucune régénération de l'angle de contact n'est observée dans cet essai (Fig. 4C). Ces résultats confirment donc que l'augmentation de l'angle de contact après immersion des bétons non carbonatés dans l'eau de mer est uniquement due au développement du biofilm sur leurs surfaces.

\section{Conclusions}

Les résultats confirment que la carbonatation de la surface joue un rôle primordial dans l'initiation de la biocolonisation du béton : les échantillons de béton carbonaté sont plus bioréceptifs que ceux non carbonatés aux jours primaires ce qui est conforme à la littérature [45].
La phase de latence est plus courte et la cinétique de colonisation est plus rapide dans le cas d'échantillons carbonatés. La colonisation de la surface pourrait protéger le matériau en augmentant l'hydrophobicité du matériau et en diminuant ainsi l'absorption d'eau contenant des substances agressives. Une fois le biofilm fixé à la surface, il modifie les propriétés physicochimiques telles que le $\mathrm{pH}$ et l'hydrophobicité de la surface ce qui pourra faciliter ensuite l'adhésion d'autres organismes tels que les champignons, les microalgues, les macroalgues et les invertébrés formant ainsi un biofouling $[14-16]$.

Ce type de tests pourrait potentiellement être utilisé pour évaluer la bioréceptivité d'un matériau cimentaire destiné à être immergé dans l'eau de mer. Cette évaluation de la bioréceptivité pourrait être utilisée pour sélectionner des matériaux pour l'écoconception des structures maritimes. Pour valider cette configuration de test de laboratoire, nous devons vérifier si ces tests donnent des résultats comparables avec des tests de biocolonisation qui seront effectués dans des conditions naturelles (in situ). 


\section{References}

1. S. Dupray, G. Robertshaw, N. Bean, K. Gamst, L'utilisation du béton pour les ouvrages maritimes: synthèse francoanglaise des bonnes pratiques, in: $\mathrm{X}^{\mathrm{es}}$ Journées, Sophia Antipolis, Editions Paralia, 2008, pp. 719-728, https://doi. org $/ 10.5150 /$ jngcgc.2008.068-D

2. M. Sosa, T. Pérez-López, J. Reyes, F. Corvo, R. CamachoChab, P. Quintana, D. Aguilar, Influence of the marine environment on reinforced concrete degradation depending on exposure conditions, Int. J. Electrochem. Sci. 6, 19 (2011)

3. C. Munzer, Étude de l'action d'un bioadjuvant aux substances extracellulaires sur la microstructure et les caractéristiques de surface de pâtes cimentaires pour des bétons plus éco-respectueux, $\mathrm{PhD}$ Thesis, Université de Strasbourg, 2016

4. K.B. Ahmed, Étude de l'encrassement biologique de matériaux cimentaires en eau de rivière: analyse de l'influence des paramètres de surface des pâtes cimentaires, PhD Thesis, Université de Strasbourg, 2016. https://tel. archives-ouvertes.fr/tel-01544842/document (accessed September 27, 2018)

5. V. Georges, S. Roux, F. Feugeas, A. Lecomte, Colonisation biologique de pâtes de ciment exposées à une eau douce naturelle, Matériaux \& Techniques 105, 204 (2017), https: / doi.org $/ 10.1051 / \mathrm{mattech} / 2017030$

6. S. Dobretsov, H.-U. Dahms, P.-Y. Qian, Inhibition of biofouling by marine microorganisms and their metabolites, Biofouling 22, 43-54 (2006)

7. N. Fusetani, Biofouling and antifouling, Nat. Prod Rep. 21, 94-104 (2004)

8. H.-C. Flemming, G. Schaule, T. Griebe, J. Schmitt, A. Tamachkiarowa, Biofouling the Achilles heel of membrane processes, Desalination 113, 215-225 (1997)

9. N.M. Farhat, L. Javier, M.C.M. Van Loosdrecht, J.C. Kruithof, J.S. Vrouwenvelder, Role of feed water biodegradable substrate concentration on biofouling: Biofilm characteristics, membrane performance and cleanability, Water Res. 150, 1-11 (2019), https://doi.org/10.1016/j. watres.2018.11.054

10. S. Dobretsov, R.M.M. Abed, M. Teplitski, Mini-review: Inhibition of biofouling by marine microorganisms, Biofouling 29, 423-441 (2013), https://doi.org/10.1080/08927014.2013. 776042

11. L.V. Evans, Structure and function of biofilms, in: Biofilms, CRC Press, 2000, pp. 16-33

12. P. Stoodley, K. Sauer, D.G. Davies, J.W. Costerton, Biofilms as complex differentiated communities, Ann. Rev. Microbiol. 56, 187-209 (2002)

13. M. Hayek, C. Baraquet, R. Lami, Y. Blache, M. Molmeret, The Marine Bacterium Shewanella woodyi Produces C8-HSL to Regulate Bioluminescence, Microb Ecol. (2019), https:// doi.org/10.1007/s00248-019-01454-z

14. M. Salta, J.A. Wharton, Y. Blache, K.R. Stokes, J.-F. Briand, Marine biofilms on artificial surfaces: structure and dynamics, Environ. Microbiol. 15, 2879-2893 (2013), https://doi.org/10.1111/1462-2920.12186

15. F. Brian-Jaisson, A. Ortalo-Magné, L. Guentas-Dombrowsky, F. Armougom, Y. Blache, M. Molmeret, Identification of bacterial strains isolated from the Mediterranean Sea exhibiting different abilities of biofilm formation, Microb. Ecol. 68, 94-110 (2014), https://doi.org/10.1007/s00248013-0342-9
16. J.-F. Briand, X. Pochon, S.A. Wood, C. Bressy, C. Garnier, K. Réhel, F. Urvois, G. Culioli, A. Zaiko, Metabarcoding and metabolomics offer complementarity in deciphering marine eukaryotic biofouling community shifts, Biofouling 34, 657672 (2018), https://doi.org/10.1080/08927014.2018.1480757

17. M. Sanchez-Silva, V. Rosowsky David, Biodeterioration of construction materials: state of the art and future challenges, J. Mater. Civil Eng. 20, 352-365 (2008), https://doi.org/ 10.1061/(ASCE)0899-1561(2008)20:5(352)

18. S. Roux, F. Feugeas, A. Cornet, Biodégradation des bétons : analyse des bétons et mortiers en contact avec une eau douce naturelle, Matériaux \& Techniques. 93, 123 (2005)

19. S. Wei, Z. Jiang, H. Liu, D. Zhou, M. Sanchez-Silva, Microbiologically induced deterioration of concrete-A review, Braz. J. Microbiol. 44, 1001-1007 (2014), https:// doi.org/10.1590/S1517-83822014005000006

20. B.T. Patil, M.R. Gajendragad, G. Ranganna, A.B. Wagh, T. Sudhakaran, Impact of biofouling on corrosion resistance of reinforced concrete, (1994), http://agris.fao.org/agris-search/ search.do?recordID =AV20120139400 (accessed August 22, 2019)

21. S. Soleimani, B. Ormeci, O.B. Isgor, Growth and characterization of Escherichia coli DH5 $\alpha$ biofilm on concrete surfaces as a protective layer against microbiologically influenced concrete deterioration (MICD), Appl. Microbiol. Biotechnol. 97, 1093-1102 (2013)

22. S. Perkol-Finkel, I. Sella, Ecologically active concrete for coastal and marine infrastructure: Innovative matrices and designs, in: From sea to shore? Meeting the challenges of the sea, ICE Publishing, 2014, pp. 1139-1149, https://doi.org/ $10.1680 /$ fsts. 59757.124

23. M.A. Coombes, H.A. Viles, L.A. Naylor, E.C. La Marca, Cool barnacles: Do common biogenic structures enhance or retard rates of deterioration of intertidal rocks and concrete?, Sci. Total Environ. 580, 1034-1045 (2017), https://doi.org/ 10.1016/j.scitotenv.2016.12.058

24. T. Chlayon, M. Iwanami, N. Chijiwa, Combined protective action of barnacles and biofilm on concrete surface in intertidal areas, Construct. Build. Mater. 179, 477-487 (2018), https://doi.org/10.1016/j.conbuildmat.2018.05.223

25. F. Bartoli, A.C. Municchia, Y. Futagami, H. Kashiwadani, K.H. Moon, G. Caneva, Biological colonization patterns on the ruins of Angkor temples (Cambodia) in the biodeterioration vs bioprotection debate, Int. Biodeterior. Biodegrad. 96, 157-165 (2014), https://doi.org/10.1016/j.ibiod.2014.09.015

26. S. Pande, S. Shitut, L. Freund, M. Westermann, F. Bertels, C. Colesie, I.B. Bischofs, C. Kost, Metabolic cross-feeding via intercellular nanotubes among bacteria, Nat. Commun. 6, (2015), https://doi.org/10.1038/ncomms7238

27. C. Lors, F. Feugeas, B. Tribollet, Interactions matériauxmicroorganismes: bétons et métaux plus résistants à la biodétérioration, EDP Sciences, 2017

28. A. Dubosc, Étude du développement de salissures biologiques sur les parements en béton: mise au point d'essais accélérés de vieillissement, $\mathrm{PhD}$ Thesis, Toulouse, INSA, 2000

29. E. Dalod, Influence de la composition chimique de mortiers sur leur biodétérioration par les algues, PhD Thesis, École Nationale Supérieure des Mines de Saint-Etienne, 2015, https://tel.archives-ouvertes.fr/tel-01148160/document (accessed October 9, 2018)

30. J.-C. Souche, S. Pioch, M. Salgues, K.D. Weerdt, A. Agostini, M. Hayek, De la conception à l'éco-conception des ouvrages maritimes: intégrer la nature au projet d'aménagement 
maritime, Revue Paralia. 12, (2019), https://doi.org/ 10.5150/revue-paralia.2019.n01

31. J.-C. Souche, G. Le Saout, M. Salgues, S. Pioch, Effets de bétons bio-actifs sur la colonisation marine en environnement méditerranéen, Matériaux \& Techniques 104, 504 (2016)

32. T.H. Tran, A. Govin, R. Guyonnet, P. Grosseau, C. Lors, E. Garcia-Diaz, D. Damidot, O. Devès, B. Ruot, Influence of the intrinsic characteristics of mortars on biofouling by Klebsormidium flaccidum, Int. Biodeterior. Biodegrad. 70, 31-39 (2012), https://doi.org/10.1016/j.ibiod.2011.10.017

33. T.H. Tran, A. Govin, R. Guyonnet, P. Grosseau, C. Lors, D. Damidot, O. Devès, B. Ruot, Influence of the intrinsic characteristics of mortars on their biofouling by pigmented organisms: Comparison between laboratory and field-scale experiments, Int. Biodeterior. Biodegrad. 86, 334-342 (2014), https://doi.org/10.1016/j.ibiod.2013.10.005

34. A. Govin, T.H. Tran, R. Guyonnet, P. Grosseau, C. Lors, D. Damidot, O. Devès, B. Ruot, Ability in biofouling by Klebsormidium flaccidum of mortars: Influence of the intrinsic characteristics, in: First International Conference on Concrete Sustainability (ICCS), 2013, pp. 914-919

35. O. Guillitte, Bioreceptivity: a new concept for building ecology studies, Sci. Total Environ. 167, 215-220 (1995), https://doi.org/10.1016/0048-9697(95)04582-L

36. D.J. Giannantonio, J.C. Kurth, K.E. Kurtis, P.A. Sobecky, Effects of concrete properties and nutrients on fungal colonization and fouling, Int. Biodeterior. Biodegrad. 63, 252-259 (2009), https://doi.org/10.1016/j.ibiod.2008.10.002

37. A.Z. Miller, P. Sanmartín, L. Pereira-Pardo, A. Dionísio, C. Saiz-Jimenez, M.F. Macedo, B. Prieto, Bioreceptivity of building stones: A review, Sci. Total Environ. 426, 1-12 (2012), https://doi.org/10.1016/j.scitotenv.2012.03.026

38. C. Munzer, E. Belhaj, T. Meylheuc, A. Lecomte, F. Feugeas, Effets d'un bioadjuvant sur les caractéristiques de surface de pâtes cimentaires, Matériaux \& Techniques 103, 208 (2015), https://doi.org/10.1051/mattech/2015024

39. C. Grant, Fouling of terrestrial substrates by algae and implications for control-A review, Int. Biodeterior. Bull. 18, 57-65 (1982)

40. K.M. Dooley, F.C. Knopf, R.P. Gambrell, Ph-neutral concrete for attached microalgae and enhanced carbon dioxide fixation-Phase I, Federal Energy Technology Center, Morgantown, WV (US), Federal Energy Technology Center, Pittsburgh, PA (US), 1999, https://doi.org/ 10.2172/778919

41. B.P. Guilbeau, F.P. Harry, R.P. Gambrell, F.C. Knopf, K.M. Dooley, Algae attachment on carbonated cements in fresh and brackish waters-Preliminary results, Ecol. Eng. 20, 309-319 (2003), https://doi.org/10.1016/S0925-8574(03) 00026-0

42. B. Prieto, B. Silva, O. Lantes, Biofilm quantification on stone surfaces: comparison of various methods, Sci. Total Environ. 333, 1-7 (2004), https://doi.org/10.1016/j.scito tenv.2004.05.003

43. G.D. Bixler, B. Bhushan, Biofouling: Lessons from nature, Philos. Trans. Royal Soc. A: Mathemat. Phys. Eng. Sci. 370, 2381-2417 (2012), https://doi.org/10.1098/rsta.2011.0502

44. H. Barberousse, Étude de la diversité des algues et des cyanobactéries colonisant les revêtements de façade en France et recherche des facteurs favorisant leur implantation, 2006.

45. E. Dalod, A. Govin, R. Guyonnet, P. Grosseau, C. Lors, D. Damidot, Influence of the chemical composition of mortars on algal biofouling, in: K.L. Fentiman, C.H. Mangabhai, R.J. Scrivener (Eds.), International Conference on Calcium Aluminates, IHS BRE Press, Palais des Papes, Avignon, France, 2014 pp. 523-534, https://hal.archives-ouvertes.fr/ hal-01053366 (accessed October 16, 2018)

Citation de l'article : Mahmoud Hayek, Marie Salgues, Frédéric Habouzit, Sandrine Bayle, Jean-Claude Souche, Klartjee De Weerdt, Sylvain Pioch, L'influence de la carbonatation sur la biocolonisation de matériaux cimentaires dans le milieu marin, Matériaux \& Techniques 108, 202 (2020) 